\title{
An Auto Lighting Device for Backlight System Detection
}

\author{
Jiaxing Ye ${ }^{1}$,ShuangxiWang ${ }^{1, a}$, Zhang Dan $^{1}{ }^{1}$ RuitaoXie $^{2}$, Xiao Yin ${ }^{2}$ \\ ${ }^{1}$ College of Engineering, Shantou University, Shantou, 515063, Guangdong, China; \\ ${ }^{2}$ Shantou Institute for Light IndustrialEquipment, Shantou, 515060, Guangdong, China \\ aemail: sxwang@stu.edu.cn
}

\begin{abstract}
Keywords: Backlight system; Qualitydetection; Auto lighting device; FPC electrode; Gold finger
\end{abstract}
\begin{abstract}
In the manufacturing of electronic product, backlight quality always affects the final rate of good product. To date, the backlight qualityis usually detected by manual eyes.In this article,an auto lighting device used forbacklightsystemdetectionwasfabricatedwith STM32 chip which basedon the ARM core. Firstly,the FPC was pressed closely on the dense goldfinger.Then, positive or negative weak current signal was sent respectively fromthe transistor array which was controlled by the STM32 chip. Thirdly, the positive or negative of FPC electrode wasjudged intelligently by the STM32 chip.Finally, according to the judgment results, the operating power with corresponding electrode polaritywas provided tolight the backlight system. The auto lighting detection of the backlight quality could be realized perfectly by this device.
\end{abstract}

\section{Introduction}

In recent years, the popularity of intelligent mobile has promoted the rapid development of mobile phone LCD screen. It is reported by NPD Display Search [1]that, in the first half of 2014, the output of mobile phone LCD screen in China was over than 300 million pieces. Generally, the LCD screen consists of backlight unit, liquid crystal screen and lenticular raster system [2]. As the core part of LCD screen, the productivity of backlight unit has heavy influence on the shipment of LCD screen.

In order to ensure the quality of electronic product during the production process of backlight unit, it needs to inspect the backlight unit one by one to sort out theunqualified products. Some kinds ofdefectsin backlight unit need to be inspected under the conditions of lighting.In the manual inspection,the worker needs to position the backlight unit on the fixture mechanically and ensures the correctness of the FPC electrodepolarity which is on the top of backlight unit. Then, aligns the electrode polarity of FPC on the fixture and presses them by hand. At last, the worker lights the backlight unit and inspects the quality. Obviously,the efficiencyof manual operation is very poor andthe test standard is hard to unify. Facing withthe pressure of hugedemandof products and shortage of labor force in electronic manufacturing industry, the machine vision equipment has been developed to improve the efficiency and quality of the production[3][4]. In fact, the key technology of thebacklightunit inspectiondeviceis how to light the backlight unitautomatically and quickly.Due to the mechanical manufacture deviation, there are printed deviation and registration deviation with FPC electrode on the backlight unit. If utilizing traditional counterpoint lighting method in automatic detection equipment, there would be some possibility that FPC electrode connects with the positive and negative electrode of fixture at the same time, which would result in the good product to be misjudged as NG product. It will seriously affect the detection results and detection efficiency of the inspection equipment.Therefore, it is urgently requiredto invent anefficientautomatic lightingdevice.

Inthis research,an automatic lightingdevicehas been developedthrough the analysis of the traditionalmanual lightinginspection. The connect mode of traditional lighting device is replaced by the connection between FPC electrode anddense goldfingerwhich contains numerous tiny electrodefilaments. The polarity of electrodefilament can be set intelligently according to the detected polarity of FPC electrode on the backlight unit.In this way, the contactdeviationresultedfrom mismatch between FPC electrode and dense golden finger electrode 
of the fixture can be ignored.It is equivalent tothat the electrode polarity of electrodefilament can moveforward according to the polarity of FPCelectrode. Experiment results showed thatdifferenttypes of backlight unit can belighted by the auto lighting device fast and accurately.

\section{Manual lighting inspection}

The traditional lighting inspection device consists offixture and power supply system.During the inspection of backlight unit, the workerneeds to fix the backlight unit on the fixture mechanically and make sure the FPC electrode and the electrodes on the fixture arealigned,showed in Fig.1(a). Then, inspects the quality while pressing the FPC electrode and lighting the backlight unit up. In fact, thereare twomain problems of the traditional lightinginspection.

(1) Poor universality.One type of the fixture of traditional light device is onlysuitablefor certain types of FPC electrode.If the FPC electrode typeof backlight unit has changed, it needs to change the fixture type at the same time, which will raise the processing cost.

(2)Easy to misjudge. In testingprocess,the stress and the alignmenterror betweenFPC electrode and electrode filamentare controlled by workers.Working in high-tension condition, it is difficult to ensure the accuracy and consistency of the inspectionthatwouldlead to misjudge of the backlight qualitybecause the backlight unit couldn’t be lighted.

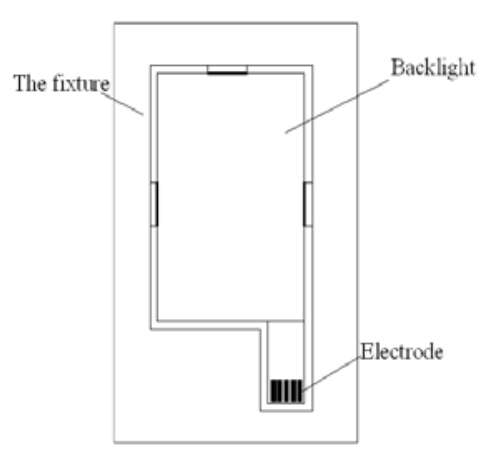

(a)Electrodes alignment

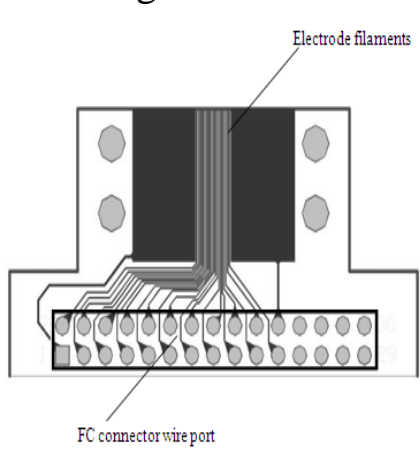

(b)PCB of gold finger

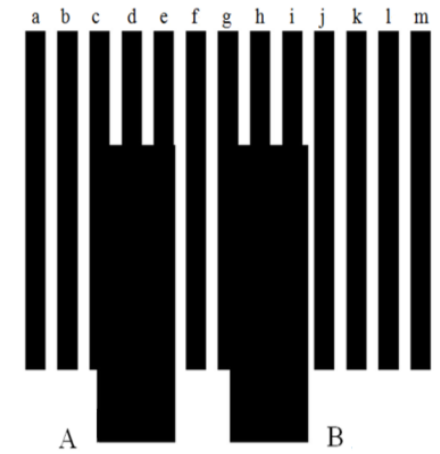

(c)Contact model

Fig.1The layout of electrodes

\section{The working principle of the auto lighting device}

On the background of increasing backlight output and high scale of production automation, it's a general trend to apply the on-line automatic detection equipment. However, there is printed deviation of FPC electrodedue to mechanicalmanufacture deviation.If directlyadopting the traditional counterpoint lighting method in the on-line automatic detection equipment, it is very possible tolead tothe trouble that FPC electrode contacts with wrong electrode of fixture.In this situation, the backlight unitcouldn't be lighted normally.

Fig.2 shows the system construction of auto lighting device, including densegold finger,FCconnector wire, intelligent control system, DC stabilized power supply and the encapsulation shell. The intelligent control system includesgoldfingerconnector, transistor array, main control panel and control power supply.

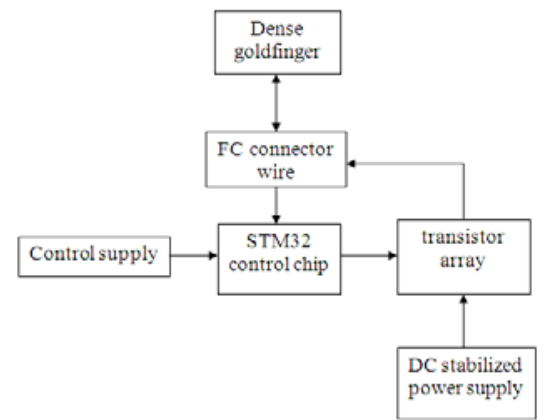

Fig.2Scheme of auto lighting device 
Fig.1(b)shows the dense goldfinger.It can be seen that the densegold fingercomprises many tiny electrode filaments, every electrode filaments connect withtwo DTR drive transistor which areincluded in the transistor array independently.

The working principle of the auto lighting device is as follows.Firstly,pressthe FPC electrode on the densegold fingerclosely and ensure that at least one electrode filament of gold finger contact with the FPC electrode.Secondly, the dense gold finger connectswith the intelligent control systemthroughFC connector wire. Positive or negative weak current signal is sent rapidlyand orderlyfromSTM32 controlchipwhich is located on main controller boardto the dense gold finger.Throughtransistor array and sampling circuit, the intelligent control system can recognizeautomaticallywhere the FPC electrode are situated on the gold fingerand judge the polarity relationship betweengold finger electrode filaments and FPC electrode. Thirdly, according to the judgmentresults, the intelligent control systemclassifiesthe electrode filament into twocategories (anode and cathode)and find out the boundarybetween anode and cathode of electrode filament. At last, the operating power is providedwith working voltage or working currenttolight the backlight unit.

Fig.1(c)shows the contact model of FPC electrode and dense gold finger.There are two electrodes in the FPC.Arepresentspositive electrode and Brepresentsnegative electrode. There are 13 electrode finnyfilaments on the gold fingerwhich are tagged with letters from a to $\mathrm{m}$.TheA electrode contactswith the electrode filaments marked with c,d,e.The B electrode contacts with the electrode filaments marked withg,h, i.

Take the electrode type of FPC (showed in Fig.1 (c) as an exampleto explain the detecting principle. Firstly, set the intervalnumberasthreewith the controlprogram before inspecting. Then, STM32 control chipcontrols transistor arraytry to transmit weak positive and negative signals to gold finger sequentially and quickly. It can be seen that the electrode filament can't connect with FPC electrode to form the current loop while electrode-a flows through positive current and electrode-e flows through negativecurrent. And the same situation will happen if electrode-b flows through positive current and electrode-f flows through negative current. The current circuit will be achieved until electrode-c flows through positive current and electrode-g flows throughnegativecurrent.Atthe same time, the sampling circuitreceived the signal and feeds back to the STM32control chip. TheSTM32 control chip judgesthat if the electrode-c filamentcontactedwith the positive FPC electrode and the electrode-gfilamentcontactedwith the negative FPC electrode or not. When the current loopis formed, the negative signal on electrode filament will keep the samewhilethe positive signal moving forward.Then, theelectrode-g filament keeps negative signaland electrode-dflowsthrough the positive signal. By such analogy,the positive signal keepsmoving,until electrode-f flowsthroughthe positive signaland the current loop is destroyed simultaneously. Therefore, the intelligent control system is able to determine that electrode-ffilament of densegold finger is the boundary between anode and cathode of electrode filamentsrelative to FPC electrode.At last, the operating power was provided tolight the backlight unit up with corresponding electrode polarityby controlling the transistor array.Under the control of STM32 control chip, the positive charge flows through electrodes from a to eand the negative charge flows through electrodes from g to m while electrode-f withoutpower supply.

\section{The fabrication of auto lighting device}

According to the principle and method above, the auto lighting device has been fabricate.Fig.3shows the PCB board of control system. The STM32 control chip works as the core of control system which is exploited by STMicroelectronics. The STM32 control chiphas powerfulextending functionwith ARM as the core architecture and16kb RAM and 4kb ROM[5], which totallysatisfy the design requirement of auto lighting device. The densegold fingeris one of the most important parts in thedevice.It serves as interface channeland electric channelto connect the backlight unit with the FPC electrode. The densegold finger includes a lot of tiny electrode filaments. Every electrode filament connects the control system by FC connector wire. The inspectsignal and voltaic signal is sent from transistor arrayto FPC electrode under the control of 
STM32. The power of control system and backlight unit is supplied by power management systemseparately.

Through applying auto lighting device in backlight inspection, it only needs to press the FPC electrode densegold fingertightlyand ensure that the FPC electrode contactswithat least one electrode filament. Under the control of STM32 chip, transistor arraytransmits weak positive and negative signals to gold finger sequentially and quickly. According to the inspect signal fed back by sampling circuit, the STM32chip is able to find out the polarityboundary of electrode filamentrelative to FPC electrode. Base on the result above, the operating power is provided tolight the backlight unitwith corresponding electrode polarity. In the inspection, it will cause misjudgmentif two electrodefilaments with opposite polarity contactwith the same FPC electrode. In orderto avoid this situation,the distance between two electrode filaments with opposite polarity must have a certain interval, the interval must be larger than the width of a single FPC electrode and smaller than the total width of entireFPC.

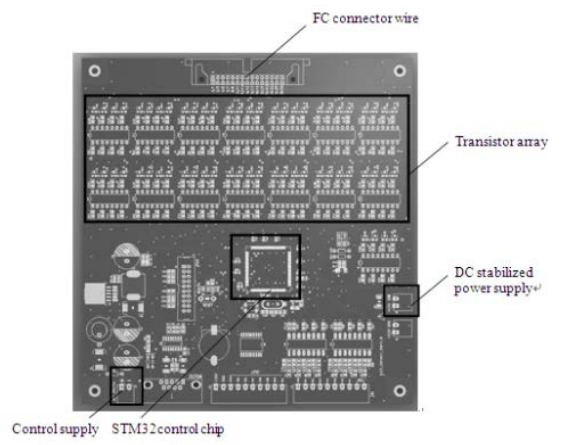

Fig.3 ThePCB board of control system

Detection resultobtainedby auto lightingdevice was shown in Table 1 .The width of electrode filament is $0.1 \mathrm{~mm}$. The intervalbetween two electrode filaments is $0.1 \mathrm{~mm}$. The size of backlight is 2.4inch. Three kinds of FPC, $(0.35 \mathrm{~mm}+0.35 \mathrm{~mm}) \times 4 \mathrm{PIN},(0.4 \mathrm{~mm}+0.4 \mathrm{~mm}) \times 5 \mathrm{PIN},(0.6 \mathrm{~mm}+0.6 \mathrm{~mm})$ $\times 5$ PIN,were chosen as the testing sample. In order to inspectthe entiretypes of FPC above, the interval between twoelectrode filaments was set as 3.Therefore, the whole width from positive electrode filament to negative electrode filament is $0.7 \mathrm{~mm}$. During the inspection test, the unlighted backlight unit needs to be inspected again bytraditional manual lighting deviceto analyze whether misjudgeexisting. Experiment results showed that there wasno misjudgment existing in two types of FPCwith reasonable preset of auto lighting device. However, the result of $\quad(0.35 \mathrm{~mm}+0.35 \mathrm{~mm})$ $\times$ 4PINFPC electrode type was non-ideal. After carefully analysis, it was found that the length of electrodes in this FPC type is too short to contact well with the densegold finger.

Table1 Testing resultsby auto lighting device

\begin{tabular}{ccccc}
\hline $\begin{array}{c}\text { The standard of FPC/ } \\
(\mathrm{mm}+\mathrm{mm}) \times \mathrm{PIN}\end{array}$ & $\begin{array}{c}\text { Test } \\
\text { number }\end{array}$ & $\begin{array}{c}\text { Lighted } \\
\text { number }\end{array}$ & $\begin{array}{c}\text { Number of } \\
\text { misjudgment }\end{array}$ & $\begin{array}{c}\text { Rate of } \\
\text { misjudgment }\end{array}$ \\
\hline$(0.35+0.35) \times 4$ & 500 & 372 & 61 & $12 \%$ \\
$(0.4+0.4) \times 5$ & 500 & 476 & 0 & 0 \\
$(0.6+0.6) \times 5$ & 1000 & 927 & 0 & 0 \\
\hline
\end{tabular}

\section{Conclusions}

In this research, the densegold finger which includes many electrode filaments has been taken as the contact mediumto link up the auto lighting deviceand backlight unit.The polarity of every electrodefilament can be set by the pair-shareelectrode of backlight unit intelligently.Itis equivalent tothe mechanism that the electrodefilament can be moved according to the polarity of FPCelectrode. In this way, the contact deviationresulted from mismatch between FPC electrode and dense golden finger electrode can be ignored. Experiment results showed that the backlight unit with different types of FPC could be lighted quickly and accurately with the same dense gold finger. The auto lighting device hasstrong adaptability and accuracy in backlightsystemdetection. 


\section{Acknowledgement}

This research wassupported by Scientific Research Funding of Shantou University (NTF12001), and Science and Technology Project of Shantou City (No.2013-26)

\section{References}

[1] DisplaySearch[OL].http://www.displaysearch.com/cps/rde/xchg/displaysearch/hs.xsl/news.asp

[2] Lin ChenSheng, Wu WeiZun, Lay YunLong.A digital image-based measurement system for a LCD backlight module[J]. Optics \& Laser Technology, 2001, 33(7):499-505.

[3] ZaunerG.Machine vision based quality inspection of flat glass products [J].The International Society for Optical Engineering, 2014, 90(24):24-28.

[4] Zhang Zhongjian, He yongyi, Li Jun.An application of machine vision in automatic mobile-phone lens assembly equipment[J].Applied Mechanics and Materials, 2012,130-134: 3543-3547

[5] STM32 datasheet[OL].http://www.stmicroelectronics.com.cn/web/cn/home.html 